\title{
The role of the perception of family environment in relation to body dissatisfaction, disordered eating and difficulties in close relationships
}

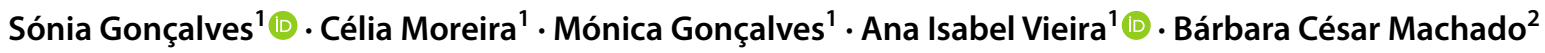

Received: 26 June 2018 / Accepted: 18 July 2018 / Published online: 31 July 2018

(c) Springer Nature Switzerland AG 2018

\begin{abstract}
Purpose Invalidating childhood environments have been characterised as those in which caregivers do not validate a child's personal experiences and the communication of emotions is considered inappropriate, being either ignored or punished. The current study assessed the relationships among invalidating childhood environments, body dissatisfaction, disordered eating and difficulties in close relationships in a sample of college students.

Methods In this study, 362 non-clinical participants with ages ranging from 17 to 25 years $(M=20.02, \mathrm{SD}=1.51)$ completed self-report measures.

Results Difficulties in close relationships, body dissatisfaction and disordered eating were significantly higher in invalidating families than in validating families. Invalidating childhood environments were associated with higher body dissatisfaction and disordered eating, and both associations were mediated by difficulties in close relationships. Finally, body dissatisfaction mediates the effects of BMI and difficulties in close relationships on eating disorder symptoms.

Conclusions Perceived experiences of invalidation in childhood may be associated with eating disorder symptomatology. In addition, difficulties in close relationships during adulthood possibly contribute to the emergence of eating disorder symptoms.

Level of Evidence Level V, descriptive study.
\end{abstract}

Keywords Invalidating childhood environments · Disordered eating · Body dissatisfaction · Difficulties in close relationships

\author{
Sónia Gonçalves \\ sgoncalves@psi.uminho.pt \\ Célia Moreira \\ celiasofiamoreira@gmail.com \\ Mónica Gonçalves \\ monica_rcg93@hotmail.com \\ Ana Isabel Vieira \\ anavieira.psi.uminho@gmail.com \\ Bárbara César Machado \\ bcmachado@porto.ucp.pt \\ 1 Psychotherapy and Psychopathology Research Unit, CIPsi, \\ School of Psychology, University of Minho, Campus de \\ Gualtar, 4710-057 Braga, Portugal \\ 2 CEDH, Centre for Studies in Human Development, \\ Faculty of Education and Psychology, Catholic University \\ of Portugal, Porto, Portugal
}

\section{Introduction}

Early parent-child relationships are widely conceptualised using two major constructs: attachment theory [1] and parental bonding [2]. Briefly, the first theorises that children respond to a caregiver's behaviour to effectively achieve care and security. The second hypothesises that parenting contributes to bonding by providing care and protection [3].

The family environment during childhood is a relevant factor for understanding parent-child relationships. In particular, invalidating experiences during childhood have raised the attention of clinicians and researchers. Invalidating childhood environments have been characterised by the existence of a poor fit between the environment and the child's temperament, where the child's personal experiences are not validated by caregivers, and where the communication of emotions is considered as inappropriate, being either ignored or punished [4-6]. Individuals raised in invalidating families learned to self-invalidate their emotions because 
they were not allowed to show negative emotions during childhood.

Interestingly, research studies have associated invalidating family contexts with eating psychopathology. In fact, according to Linehan's model [5], individuals who grew up in invalidating families are more likely to cope with their negative emotional experiences via non-adaptive behaviours such as impulsive eating or maintaining a restrictive diet and engaging in excessive physical exercise $[4,5,7]$. Research has consistently shown that dysfunctional family environments are a risk factor for emotional dysregulation, which in turn can contribute to the emergence of eating disorders [7-10]. In line with this evidence, in the study of Haslam, Arcelus, Farrow, and Meyer [11], with a non-clinical sample, positive associations were found between the experience of maternal and paternal invalidation and eating disorderrelated attitudes.

Previous research has also evidenced the influence of family environments on the body satisfaction. For instance, Boutelle, Eisenberg, Gregory, and Neumark-Sztainer [12] conducted a longitudinal study and found that a healthy connectivity between parents and children was associated with greater body-image satisfaction in a non-clinical sample of adolescent girls. Another study compared young adults from different childhood family environments and verified that the participants having grown up in invalidating families required greater external validation to achieve a more positive self-view, as well as demonstrated higher insecurity with their body image [13].

Therefore, invalidating family environments were associated with both eating disorder symptoms and body dissatisfaction. The relationship between these three variables is strengthened by other studies that describe higher levels of body dissatisfaction to greater severity of eating symptomatology [14-16].

Finally, family environments were also associated with adult relationships. A recent study by Fraley, Heffernam, Vicary, and Brumbaugh [17] suggested that family interactions influence the mental representations of the self, which in turn influence adult relationships (e.g., friends and partners). Moreover, research has also shown that attachment relationships, either in childhood or adulthood, affect the body image perception and satisfaction $[13,14,18]$.

Difficulties in establishing close relationships have been widely related to eating psychopathology. Tasca et al. [19] considered a clinical sample of women exhibiting eating disorder symptomatology and found high levels of insecure attachment. In other studies, women from community samples with eating disorders symptoms presented characteristics similar to insecure and anxious attachment styles in adult intimate relationships [20]. Arcelus et al. [14] conducted a meta-analysis and obtained an association between subclinical eating symptoms and difficulties in intimate relationships during adulthood. In the same way, a study of Japanese women found that more than half of the sample developed an eating disorder after the occurrence of marital problems, the end of a relationship, or a divorce [21]. Other studies have also linked lower levels of support from romantic partners to an increased eating disorders symptomatology $[14,22]$. In particular, Kiriike et al. [21] argued that marital stress was considered as the precipitating factor in most cases of eating disorders and consistently, improvements in eating disorder symptoms were often connected with happier feelings in close relationships.

In sum, many studies have found associations between invalidating family relationships, body dissatisfaction, eating disorder symptoms, and difficulties in close relationships. However, to the best of our knowledge, the potential intermediary processes that link these variables have not been investigated. For example, it is unclear how an invalidating childhood environment could contribute to the development of eating problems later in life. Possible mechanisms may come through the body dissatisfaction and the development of difficulties in close relationships. Given the scarcity of research regarding the association between these variables, this study sought to acquire a better understanding of this association in a community sample of college students.

In specific, this study has the following three main objectives. First, it aims to test if individuals who characterize their families as invalidating present greater difficulties in establishing close relationships, greater body dissatisfaction and higher levels of eating disorders symptomatology. Second, this research intends to clarify the association between all these variables by specifying a pattern of relationships between them. Moreover, since body mass index (BMI) has a clear and strong effect in body dissatisfaction and eating psychopathology, the pattern of relationships should also account for the effect of this measure. The study of these relationships maybe useful for researchers and clinicians, allowing to identify possible early origins of disordered body and eating attitudes. Finally, this study seeks to explore possible mediator effects in the specified pattern of relationships.

\section{Methods}

\section{Participants}

In this study, 362 college students with ages ranging from 17 to 25 years old $(M=20.02, \mathrm{SD}=1.51)$ were evaluated. Participants were mostly female $(n=315,87 \%)$. The sample included $223(61.6 \%)$ students from a public higher education establishment and 139 (38.4\%) students from a private higher education establishment. 


\section{Measures}

Four scales were used in this study.

Invalidating Childhood Environments Scale (ICES). The ICES $[6,23]$ consists of 14 items that evaluates maternal and paternal behaviours, defining a separate invalidating score for each parent [7]. Participants rated their experiences with each parent up to the age of 18 years old using the first 14 items on a Likert scale ranging from 1 (never) to 5 (all of the time). Items 5, 8, 12 and 14 were reverse scored. The highest average scores denote higher levels of maternal or paternal invalidation. The final four items of the measure describe three types of invalidating environment and one type of validating environment that correspond to descriptions proposed by Linehan [5]. In a chaotic family, parents are often unavailable and, in the little time that they are available, they often respond with anger to their children's demands. Thus, children are left to fend for themselves or go round to relatives and friends. In a perfect family everything is apparently perfect; however, negative emotions, such as fear and irritability, are not tolerated. Therefore, children are led to hide this type of emotions. Typical families focus on achievement and success and so, in this case, children develop cognitive control of emotions. Finally, in a validating family, parents listen to their children, show interest for their opinions, encourage their own decisions, and support them in case of difficulties. An individual might describe him- or herself as being from more than one family type. The participants were requested to respond using a Likert scale ranging from 1 ("not like my family") to 5 ("like my family all of the time"). Higher average scores denote higher levels of the family types "chaotic", "validating", "perfect" and "typical". The results of the original version of the ICES suggested acceptable levels of internal consistency in terms of maternal $(\alpha=.77)$ and paternal invalidation $(\alpha=.80)$. In this study, Cronbach's alphas were .89 and .90 for maternal and paternal invalidation, respectively.

Body Shape Questionnaire (BSQ). The BSQ [24, 25] consists of 34 items that evaluate weight and body shape concerns over the previous four weeks. Participants respond using a Likert scale ranging from 1 ("never") to 6 ("always"). Higher responses correspond to greater dissatisfaction with body image. Cronbach's alpha in this study was .98 .

Eating Disorders-15 (ED-15). The ED-15 [26] includes 15 items; 10 items concern attitudes over the past week and are divided into two sub-scales: "eating concerns" and "weight and shape concerns". These items are scored using a Likert scale ranging from 0 ("not at all") to 6 ("all the time"). The last five items assess eating behaviours over the past week, such as how many times he or she has binged or induced vomiting and the number of days during which he or she has used laxatives, exercised or restricted food intake.
In this study, Cronbach's alphas for the ED-15 ranged from .82 to .91 .

Experiences in Close Relationships Inventory (ECR). The ECR $[18,27]$ consists of 36 items assessed using a Likert scale ranging from 1 ("strongly disagree") to 7 ("strongly agree"). This measure can be used to assess close relationships based on two dimensions: "avoidance of intimacy" and "anxiety over abandonment". In the original study, this measure demonstrated acceptable levels of internal consistency with regard to the dimensions assessed. Cronbach's alphas in this study ranged from .85 to .86 .

\section{Procedure}

This study was reviewed and approved by the internal review board of the Research Center of Psychology at the University of Minho (Portugal), and it conformed to the Code of Ethics of the World Medical Association (Declaration of Helsinki) for experiments involving humans.

The participants were recruited from two educational establishments in northern Portugal. Before the measures were applied, all participants completed an informed consent document that emphasised the confidentiality of the data. The self-report measures were randomly distributed to the participants to prevent skewing the results due to fatigue when completing the questionnaires.

In this study, chaotic, perfect and typical families were considered to be invalidating families. Only individuals who described themselves as mainly belonging to the validating family type were considered from validating families.

\section{Statistical analyses}

The statistical analyses were conducted using the $\mathrm{R}$ statistical environment (Rstudio, version 3.4.3) [28]. For the variables of interest, we present a descriptive statistic (separately for validating and invalidating families) and the correlation matrix. The correlations between the variables were obtained using the "psych" package [29]. $P$ value corrections are recommended for multiple correlation analyses because multiple tests increase the probability of Type I errors. The adjusted probabilities were obtained using the Holm method.

To evaluate differences between validating and invalidating families in the main variables of interest, we created a dummy variable Group encoding the group membership information, and then performed a regression analysis which, apart from identifying between-groups differences, allowed to obtain estimates for those differences. For age and BMI, the residuals of the linear regression showed small deviations from normality, and then a robust linear regression was used in these two cases. As all the other variables are count data, generalized linear models were performed using the quasi-Poisson regression [30]. 
A path diagram model showing theoretical hypothesized relationships between the main variables was also specified. Following the recommendations of Hooper, Coughlan, and Mullen [31] and Hu and Bentler [32], we used several fit indices to evaluate model fit, including the Chi-Square statistic $\left(\chi^{2}\right)$, taking into account their degrees of freedom $\left(\chi^{2} / d f\right)$, the root-mean-square error of approximation (RMSEA), comparative fit index (CFI), Tucker-Lewis index (TLI), relative noncentrality index (RNI), goodness of fit index (GFI), adjusted goodness of fit index (AGFI) and standardized rootmean-square residual (SRMR). Finally, mediation effects were analysed. All structural equation modelling (SEM) analyses were performed using the "lavaan" $\mathrm{R}$ package [33]. In the mediation analysis, the bootstrapping method was used to compute standard errors [34, 35].

\section{Results}

\section{Associations between invalidating childhood environments, body dissatisfaction, eating disorder symptomatology and difficulties in close relationships}

Nearly $28.7 \%$ of the participants report the experience of an invalidating family environment. Furthermore, $26.7 \%$ of women report having grown up in an invalidating family compared to $42.6 \%$ of men.

The correlations between the variables of interest are presented in Table 1. Significant correlations were found between paternal invalidation and the following three variables: BMI, body dissatisfaction and weight and shape concerns. In these cases, the correlation for validating families was non-significant, but the correlation for invalidating families was significant or approached significance (BMI: $r<.01, p=1.000$ vs. $r=.29, p=.072 ; F=2.43$, $p=.02$; body dissatisfaction: $r=.08, p=1.000 ; F=2.16$, $p=.03$; weight and shape concerns: $r=.09, p=1.000$ vs. $r=.36, p=.006 ; F=2.32, p=.02)$. Regarding correlations involving the maternal invalidation, only one significant difference between validating and invalidating contexts was observed: the correlation with BMI, which is nonsignificant for validating families but is significant for invalidating families $(r=.02, p=.997$ vs. $r=.33, p=.001$; $F=2.62, p=.01)$. Significant differences between these familiar contexts were found in the correlation between BMI and weight and shape concerns, with invalidating families showing a stronger association between these two variables $(r=.53, p<.001$ vs. $r=.20, p=.032 ; F=3.28$, $p<.001)$.

\section{Differences between invalidating families and validating families in terms of body dissatisfaction, eating disorder symptoms and difficulties in close relationships}

Among the study variables, only BMI did not significantly differ across validating and invalidating familiar contexts. All the other variables exhibited significant differences between the two groups. The estimated amounts are presented in Table 2 and show that participants from invalidating families present higher scores in all domains. For example, considering the BSQ and comparing the total scores of two participants belonging to different contexts, the total score of the participant with an invalidating family is expected to be $14 \%$ higher than the total score of the other participant with a validating family (exp $(.13)=1.14), p=.007$.
Table 1 Correlations between the variables

\begin{tabular}{|c|c|c|c|c|c|c|c|c|}
\hline & Age & BMI & ICES $p$ & ICES $m$ & DCR & BSQ & ED1 & ED2 \\
\hline Age & - & .07 & -.01 & .02 & -.13 & -.05 & -.07 & -.11 \\
\hline BMI & & & .14 & $.17 *$ & $.16^{*}$ & $.37 * * *$ & $.32 * * *$ & $.21 * *$ \\
\hline ICE $p$ & & & - & $.76 * * *$ & $.36^{* * *}$ & $.24 * * *$ & $.26^{* * * *}$ & $.17 *$ \\
\hline $\mathrm{ICE} m$ & & & & - & $.37 * * *$ & $.23 * * *$ & $.22 * * *$ & $.18^{*}$ \\
\hline DCR & & & & & - & $.36 * * *$ & $.42 * * *$ & $.32 * * *$ \\
\hline BSQ & & & & & & - & $.85^{* * * *}$ & $.69^{* * *}$ \\
\hline ED1 & & & & & & & - & $.64 * * *$ \\
\hline ED2 & & & & & & & & - \\
\hline
\end{tabular}

BMI body mass index; ICESp paternal invalidation; ICESm maternal invalidation; DCR difficulties in close relationships; BSQ Body Shape Questionnaire (body dissatisfaction); ED1 eating disorder: weight and shape concerns; $E D 2$ eating disorder: eating concerns

$* p<.05, * * p<.01, * * * p<.001$ 
Table 2 Differences between validating families and invalidating families

\begin{tabular}{|c|c|c|c|c|c|c|c|}
\hline \multirow[t]{3}{*}{ Variable } & \multicolumn{4}{|c|}{ Descriptive statistic (raw data) } & \multicolumn{3}{|l|}{ Model for group difference } \\
\hline & \multicolumn{2}{|c|}{$\begin{array}{l}\text { Invalidating families } \\
(n=104)\end{array}$} & \multicolumn{2}{|c|}{$\begin{array}{l}\text { Validating families } \\
(n=258)\end{array}$} & \multirow[t]{2}{*}{$\begin{array}{l}\text { Difference estimate from } \\
\text { validating to invalidating }\end{array}$} & \multirow[t]{2}{*}{ SE } & \multirow[t]{2}{*}{$p$ value } \\
\hline & Mean & SD & Mean & SD & & & \\
\hline Age & 20.29 & 1.58 & 19.91 & 1.47 & .34 & .17 & .045 \\
\hline BMI & 21.95 & 2.86 & 21.49 & 2.63 & .41 & .29 & .158 \\
\hline DCR & 111.55 & 28.18 & 98.22 & 26.80 & .13 & .03 & $<.001$ \\
\hline DCR1 & 49.06 & 16.11 & 42.43 & 16.38 & .15 & .04 & $<.001$ \\
\hline DCR2 & 62.49 & 118.34 & 55.79 & 18.07 & .11 & .04 & .002 \\
\hline BSQ & 76.35 & 34.17 & 66.88 & 28.48 & .13 & .05 & .007 \\
\hline ED1 & 8.56 & 8.8 & 6.19 & 6.88 & .32 & .12 & .007 \\
\hline ED2 & 8.43 & 5.71 & 6.78 & 5.45 & .22 & .09 & .011 \\
\hline $\mathrm{ICES} m$ & 30.84 & 9.76 & 22.61 & 6.19 & .31 & .03 & $<.001$ \\
\hline ICES $p$ & 33.14 & 12.93 & 23.41 & 6.56 & .35 & .03 & $<.001$ \\
\hline
\end{tabular}

$D C R 1$ difficulties in close relationships: avoidance of intimacy; DCR2 Difficulties in close relationships: anxiety over abandonment

\section{The role of invalidating family childhood environment in predicting the development of body dissatisfaction, eating disorder symptoms and difficulties in close relationships}

The main aim of this study was to evaluate the pattern of relationships among invalidating childhood environment, body dissatisfaction, eating disorders symptoms and difficulties in close relationships. Then, a theoreticalbased path diagram including hypothesized relationships between the main variables was specified (Fig. 1). Taking advantage of the SEM technique, we used measures from the aforementioned questionnaires to specify all constructs as latent factors (except for BMI, which is a measured variable). We noticed that the direct relationship between difficulties in close relationships and eating disorder symptoms was not initially considered; thus, it was included after the examination of significant direct effects to improve the fit of the initial model. After confirming the good fitting of the measurement model, a path diagram was re-specified. The final model exhibits an acceptable fit to the data with the following fit values: $\chi^{2}=149.58, d f=97, \mathrm{RMSEA}=.040(90 \% \mathrm{CI}=[.027$, $.052]), \mathrm{CFI}=.969, \mathrm{TLI}=.961, \mathrm{RNI}=.990, \mathrm{GFI}=.997$, $\mathrm{AGFI}=.995$, and $\mathrm{SRMR}=.042$.
Fig. 1 Model 1-SEM for the relationships among invalidating childhood environments, body dissatisfaction, eating disorder symptoms and difficulties in close relationships. All (unstandardized) path coefficients have significance $p<.01$

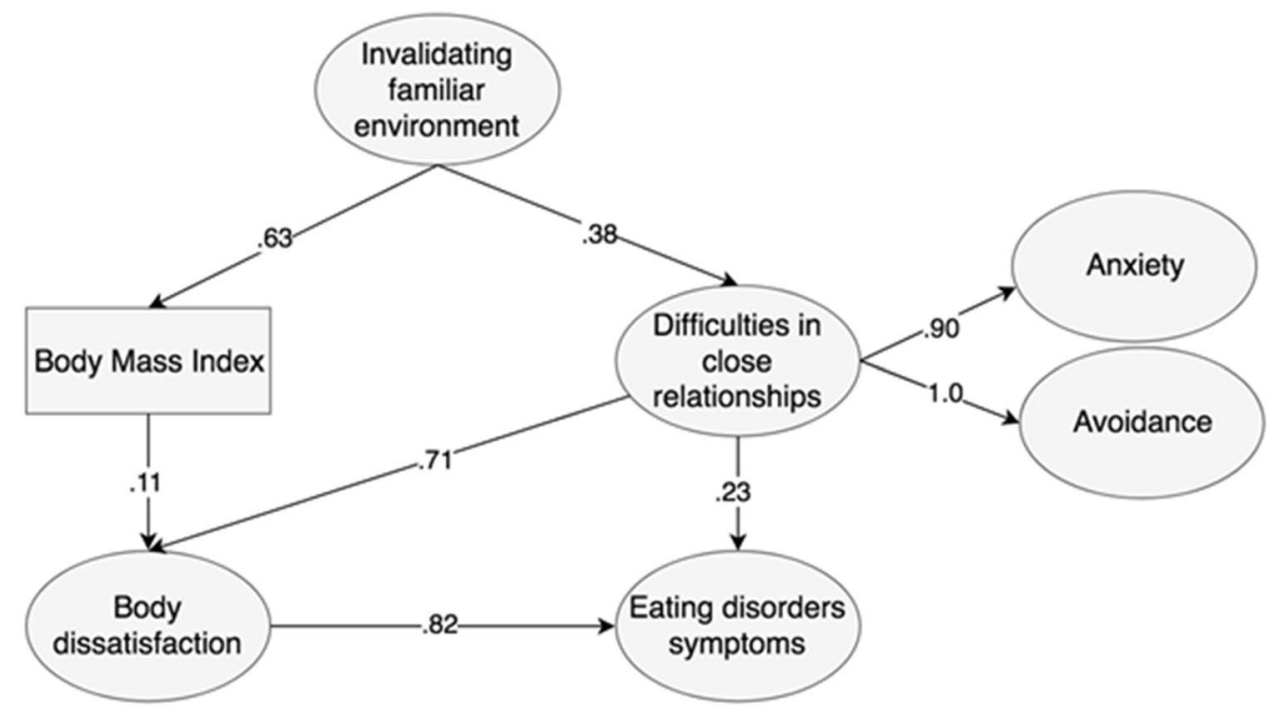


The mediation effect of difficulties

in close relationships in the association

between an invalidating childhood

environment and body dissatisfaction

and between an invalidating childhood

environment and eating disorder symptoms

We investigated the mediation effects among the major variables of Model 1 depicted in Fig. 1. The mediation models are depicted in Fig. 2, together with the estimated (unstandardised) path coefficients. Difficulties in close relationships and BMI mediated the associations between (1) invalidating childhood environments and body dissatisfaction (Model 2) and (2) invalidating childhood environments and eating disorders symptoms (Model 3). In both models, the direct and the indirect effects have opposite signs, evidencing suppression effects. Analysing the mediator effects separately, it is possible to observe that the negative sign of the direct effect is due to the variable "difficulties in close relationships", and so, this is a suppressor variable (in both models). This fact increases the importance of this mediator (suppressor), as its inclusion strengths the relationship between the independent variable and the outcomes.

\section{The mediation effect of body dissatisfaction in the relationship between an invalidating childhood environment and eating disorder symptoms and between difficulties in close relationships and eating disorder symptoms}

Body dissatisfaction mediated the relationships between (1) invalidating childhood environments and eating disorder symptoms (Model 4) and (2) difficulties in close relationships and eating disorder symptoms (Model 5).

\section{Discussion}

This research studied the relationships among invalidating childhood environments, body dissatisfaction, eating disorder symptomatology and difficulties in close relationships in a non-clinical sample of college students.

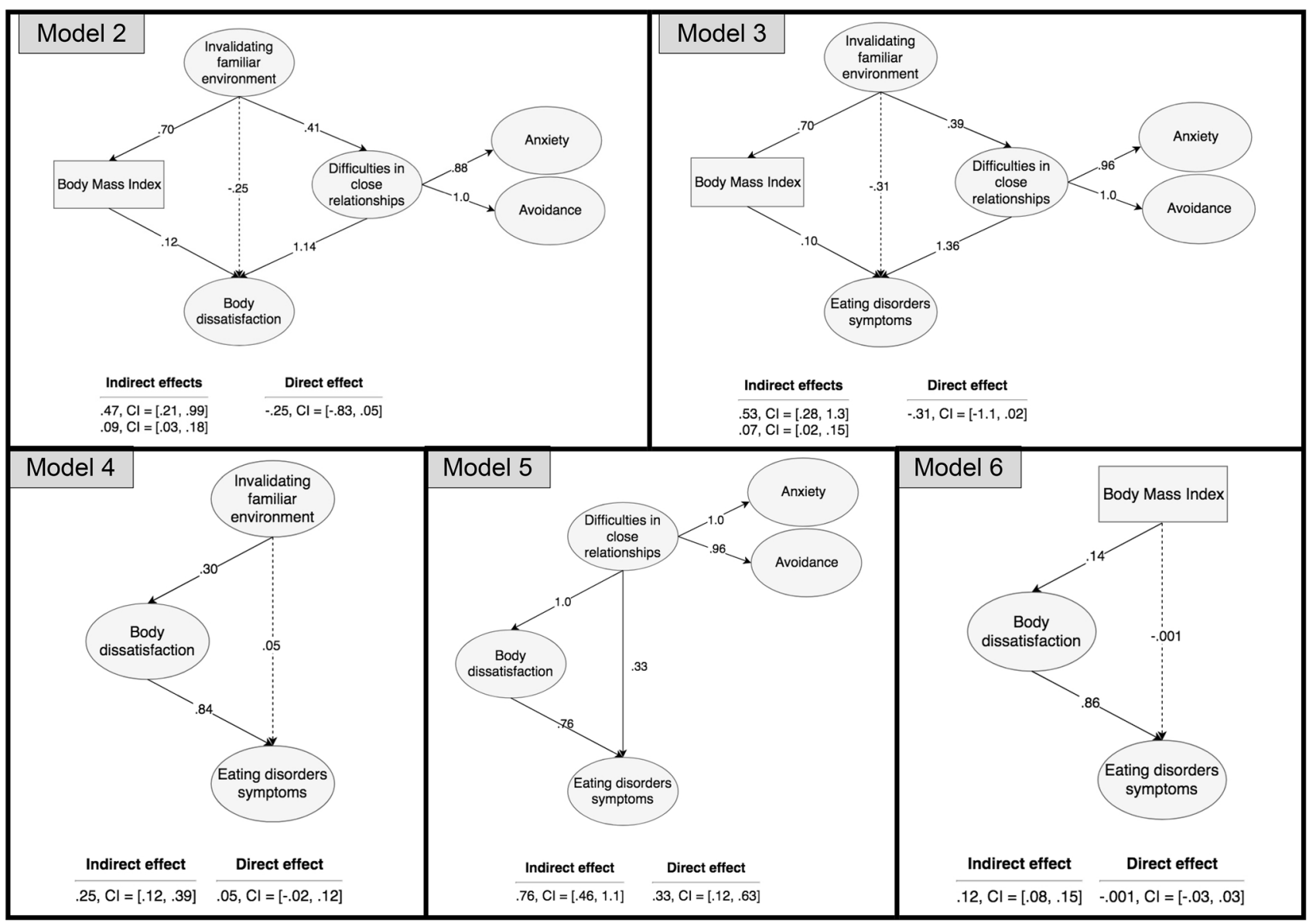

Fig. 2 Mediation effects included in Model 1. All solid lines represent significant relationships with significance $p<.01$. Dashed lines represent non-significant relationships (they evidence complete mediation). CI 95\% bootstrapped confidence intervals 
BMI, experiences of paternal and maternal invalidation, difficulties in close relationships, body dissatisfaction and eating disorder symptoms related to weight, body shape and eating concerns were positively correlated. These patterns of association are similar to those of the meta-analysis performed by Arcelus et al. [14]. These authors also verified the existence of subclinical symptoms in individuals from a community sample that explained the associations among variables such as dysfunctional family relationships, body dissatisfaction, dysfunctional eating behaviours and difficulties in establishing close romantic relationships.

Furthermore, and in the context of childhood environments, we found that difficulties in establishing close romantic relationships, body dissatisfaction and eating disorders symptoms related to weight, shape and eating concerns were much higher in invalidating than validating families. These results match the results obtained by Boutelle et al. [12] and Cheng and Mallinckrodt [13], which showed that higher levels of invalidation perceived in the family environment were associated with higher body dissatisfaction and higher levels of disordered eating. Our results are also consistent with those of Paiva and Figueiredo [18] because difficulties in parent-child emotional closeness were associated with greater difficulties in establishing close romantic relationships in adulthood. In addition, these results match those found in other studies such as Juda et al. [22] who used a non-clinical sample to find that higher levels of family invalidation were associated with greater body dissatisfaction, higher levels of eating disorders symptomatology and difficulties in establishing close relationships (defined as closeness avoidance and anxiety over abandonment).

According to Levická et al. [36], adolescent girls and young women whose family environments are not conducive to problem solving or the expression of negative emotions are more likely to develop eating disorder symptoms. Our model also supports the relationships among invalidating childhood environment, body dissatisfaction and eating disorder symptoms, thereby advancing the field and confirming that both difficulties in close relationship and BMI mediate the associations between these variables. In this sense, our results suggest that invalidating childhood experiences are associated with greater body dissatisfaction and disordered eating, and both associations were mediated by difficulties in close relationships. In turn, these difficulties are based on the experience of greater closeness avoidance and abandonment-related anxiety. These relationships can be understood through a holistic view of body image because this construct involves prior experiences that have been internalised by the individual and filter that individual's subsequent interpersonal experiences [37]. Thus, early experiences of invalidation in the family context are likely associated with later self-invalidation. This self-invalidation can manifest as, for example, the higher body dissatisfaction associated with an individual's dysfunctional eating behaviour. If we consider the role of close relationships' difficulties in avoidance and anxiety terms, then the association found might be explained through a continuous dysfunctionality in attachment relationships that begin in childhood and continue into adulthood through the individual's fear of rejection and difficulty with trusting others [19]. Thus, body image and disordered eating might be an expression of this complex interplay in which the role of interpersonal relationships, from early infancy through adulthood, must be considered.

Finally, we found that body dissatisfaction plays an important role in the relationships between invalidating childhood environments and eating disorder symptoms; difficulties in close relationships and eating disorders symptoms; and BMI and eating disorder symptoms, mediating all the three patterns. We conclude that there is a complex dynamic of relationships between early-life family contexts and later vulnerabilities with respect to body dissatisfaction and disordered eating among individuals who struggle to experience and solve difficulties in their intimate relationships.

A high level of concern with weight and body shape was evident among the participants in this study. These results emphasise the importance of paying attention to college students because they are high-risk groups for eating disorders [36]. Thus, an awareness of this issue should be raised, particularly in schools and universities, to provide support for the identification of pathological signs through a primary prevention approach targeted at these high-risk groups. Given the current results, numerous risk factors, when combined, culminate in the development of eating disorder symptomatology, which increase the complexity of this clinical condition and the difficulty in its interpretation and intervention for healthcare professionals [36].

This study has certain limitations, notably the absence of a clinical sample. Previous research has focused on the association between dysfunctional eating behaviour and close relationships, mainly in community samples, and more studies are required to investigate individuals diagnosed with eating disorders. This study was retrospective in nature and, therefore, shares the problems inherent in retrospective bias. The retrospective assessment of childhood experiences in adults is subject to inaccuracies of memory and subjectivity in reporting. However, it is important to recognize that any measure of invalidation is a measure of perceived experience. Additionally, cause-and-effect relationships cannot be determined using retrospective and cross-sectional studies. For this reason, our mediation results should be considered as preliminary. Future research would benefit from prospective studies that examine the temporal relationship between the variables and move toward a deeper understanding of the aetiology, sustained relational difficulties and symptoms of eating psychopathology. The cross-sectional design of this study is also a limitation. Future research would benefit 
from longitudinal studies that examine the temporal relationship between the variables and move toward a deeper understanding of the aetiology, sustained relational difficulties and symptoms of eating psychopathology.

In summary, the current results confirm our initial hypotheses: Associations exist among invalidating childhood environment, body dissatisfaction, eating disorders symptomatology and difficulties in close relationships. Given these results, further research on the attachment relationships of individuals with disordered eating in clinical samples is required because the current study was conducted with individuals from a non-clinical sample. Psychotherapeutic interventions should focus on parent-child emotional closeness because perceived experiences of invalidating family contexts may be associated with eating disorder symptomatology. In addition, attention must be given to these individuals' romantic relationships because difficulties in close relationships possibly contribute to the emergence of eating disorder symptoms.

Funding None.

\section{Compliance with ethical standards}

Conflict of interest On behalf of all authors, the corresponding author states that there is no conflict of interest.

Ethical approval All procedures performed in studies involving human participants were in accordance with the ethical standards of the institutional and/or national research committee and with the 1964 Helsinki Declaration and its later amendments or comparable ethical standards.

Informed consent Informed consent was obtained from all individual participants included in the study.

\section{References}

1. Bowlby J (1969) Attachment and loss: vol 1. Attachment. Basic, New York

2. Parker G, Tupling H, Brown LB (1979) Parental bonding instrument. Br J Clin Psychol 52:1-10

3. Tetley A, Moghaddam NG, Dawson DL, Rennoldson M (2014) Parental bonding and eating disorders: a systematic review. Eat Behav 15:49-59. https://doi.org/10.1016/j.eatbeh.2013.10.008

4. Ford G, Waller G, Mountford V (2011) Invalidating childhood environments and core beliefs in women with eating disorders. Eur Eat Disord Rev 19:316-321. https://doi.org/10.1002/erv.1053

5. Linehan MM (1993) Cognitive-behavioural treatment of borderline personality disorders. Guilford, New York

6. Mountford V, Corstorphine E, Tomlinsson S, Waller G (2007) Development of a measure to assess invalidating childhood environments in the eating disorders. Eat Behav 8:48-58. https://doi. org/10.1016/j.eatbeh.2006.01.003

7. Haslam M, Mountford V, Meyer C, Waller G (2008) Invalidating childhood environments in anorexia and bulimia nervosa. Eat Behav 9:313-318. https://doi.org/10.1016/j.eatbeh.2007.10.005

8. Burns EE, Fischer S, Jackson JL, Harding HG (2012) Deficits in emotion regulation mediate the relationship between childhood abuse and later eating disorder symptoms. Child Abuse Negl 36:32-39. https://doi.org/10.1016/j.chiabu.2011.08.005

9. Feinson MC, Hornik-Lurie T (2016) Binge eating \& childhood emotional abuse: a mediating role for anger. Appetite 105:487493. https://doi.org/10.1016/j.appet.2016.05.018

10. Lyke J, Matsen J (2013) Family functioning and risk factors for disordered eating. Eat Behav 14:497-499. https://doi. org/10.1016/j.eatbeh.2013.08.009

11. Haslam M, Arcelus J, Farrow C, Meyer C (2012) Attitudes towards emotional expression mediate the relationship between childhood invalidation and adult eating concern. Eur Eat Disord Rev 20:510-514. https://doi.org/10.1002/erv.2198

12. Boutelle K, Eisenberg ME, Gregory ML, Neumark-Sztainer D (2009) The reciprocal relationship between parent-child connectedness and adolescent emotional functioning over 5 years. J Psychosom Res 66:309-316. https://doi.org/10.1016/j.jpsychores .2008.10.019

13. Cheng HL, Mallinckrodt B (2009) Parental bonds, anxious attachment, media internalization, and body image dissatisfaction: exploring a mediation model. J Couns Psychol 3:365-375. https ://doi.org/10.1037/a00115067

14. Arcelus J, Yates A, Whiteley R (2012) Romantic relationships, clinical and sub-clinical eating disorders: a review of the literature. Sex Relation Ther 2:147-161. https://doi.org/10.1080/14681 994.2012.696095

15. Mantilla EF, Bergsten K, Birgegard A (2014) Self-image and eating disorder symptoms in normal and clinical adolescents. Eat Behav 15:125-131. https://doi.org/10.1016/j.eatbeh.2013.11.008

16. Shea ME, Pritchard ME (2007) Is self-esteem the primary predictor of disordered eating? Pers Individ Dif 42:1527-1537. https:// doi.org/10.1016/j.paid.2006.10.026

17. Fraley RC, Heffernan ME, Vicary AM, Brumbaugh CC (2011) The experiences in close relationships - relationship structures questionnaire: a method for assessing attachment orientations across relationships. Psychol Assess 3:615-625. https://doi. org/10.1037/a0022898

18. Paiva C, Figueiredo B (2010) Validation of the "Experiences in close Relationships"-Portuguese version. Interpersona 4:237270. https://doi.org/10.5964/ijpr.v4i2.51

19. Tasca GA, Ritchie K, Zachariades F, Proulx G, Trinneer A, Balfour L et al (2013) Attachment insecurity mediates the relationship between childhood trauma and eating disorder psychopathology in a clinical sample: a structural equation model. Child Abuse Negl 37:926-933. https://doi.org/10.1016/j.chiabu.2013.03.004

20. Evans L, Wertheim EH (2005) Attachment styles in adult intimate relationships: comparing women with bulimia nervosa symptoms, women with depression and women with no clinical symptoms. Eur Eat Disord Rev 13:285-293. https://doi.org/10.1002/erv.621

21. Kiriike N, Nagata T, Matsunaga H, Tobitani W, Nishiura T (1996) Married patients with eating disorders in Japan. Acta Psychiatr Scand 94:428-432. https://doi.org/10.1111/j.1600-0447.1996. tb09885. $\mathrm{X}$

22. Juda MN, Campbell L, Crawford CB (2004) Dieting symptomatology in women and perceptions of social support: an evolutionary approach. Evol Hum Behav 25:200-208. https://doi.org/10.1016/j. evolhumbehav.2004.02.001

23. Vieira AI, Gonçalves, M, Machado BC, Rodrigues T, Machado PPP, Brandão I et al. (2018). Psychometric properties of the Portuguese version of the invalidating childhood environment scale. Eating and Weight Disorders - Studies on Anorexia, Bulimia, and Obesity (in press)

24. Cooper PJ, Taylor MJ, Cooper Z, Fairburn CG (1987) The development and validation of the Body Shape Questionnaire. Int J Eat Disord 6:485-494. https://doi.org/10.1002/1098

25. Vieira PN, Palmeira AL, Francisco C, Teixeira PJ (2004) Validação portuguesa do Questionário de Forma Corporal. Poster 
presented at the Congresso Nacional de Psicologia da Saúde, Lisboa

26. Tatham M, Turner H, Mountford VA, Tritt A, Dyas R, Waller G (2015) Development, psychometric properties and preliminary clinical validation of a brief, session-by-session measure of eating disorder cognitions and behaviors: the ED-15. Int J Eat Disord 43:1-5. https://doi.org/10.1002/eat.22430

27. Brennan KA, Clark CL, Shaver PR (1998) Self-report measurement of adult attachment. In: Simpson JA, Rholes WS (eds) Attachment theory and close relationships. Guilford, New York, pp 46-76

28. R Development Core Team (2017) R: a language and environment for statistical computing. Vienna, Austria: R foundation for statistical computing. http://www.R-project.org/. Acessed 8 Jan 2018

29. Revelle W (2018) Psych: procedures for personality and psychological research (Version 1.8.4). Northwestern University, Illinois

30. Hilbe JM (2011) Modeling count data. In: Lovric M (ed) International encyclopedia of statistical science. Springer, Berlin, pp 108-125

31. Hooper D, Coughlan J, Mullen M (2008) Structural equation modelling: guidelines for determining model fit. EJBRM 6:53-60
32. Hu L, Bentler PM (1999) Cutoff criteria for fit indexes in covariance structure analysis: conventional criteria versus new alternatives. Struct Equ Model 6:1-55. https://doi.org/10.1080/10705 519909540118

33. Rosseel Y (2012) lavaan: an R package for structural equation modeling. J Stat Softw 48:1-36. https://doi.org/10.18637/jss. v048.i02

34. Hoyle RH (2012) Handbook of structural equation modeling. The Guilford Press, New York

35. Shrout PE, Bolger N (2002) Mediation in experimental and nonexperimental studies: new procedures and recommendations. Psychol Methods 7:422-445

36. Levická K, Kovalcíková N, Kováková L (2014) Incidence of eating disorders in family environment in high school adolescentes. Soc Behav Sci 132:391-397. https://doi.org/10.1016/j.sbspr o.2014.04.327

37. Tylka TL, Wood-Barcalow NL (2015) What is and what is not positive body image? Conceptual foundations and construct definition. Body Image 14:118-129. https://doi.org/10.1016/j.bodyi m.2015.04.001 